\title{
Image of the month: Ormond's disease: A diagnostic challenge
}

\author{
Authors: Almond Leung, ${ }^{A}$ Haitham M Elasir ${ }^{B}$ and Karim M Mahawish ${ }^{C}$
}

Idiopathic retroperitoneal fibrosis is a rare disease. Symptoms include constitutional symptoms and abdominal/flank pain. Causes of retroperitoneal fibrosis include non-infectious and infectious etiologies. Of the infectious etiologies, tuberculous aortitis is an important differential as it is associated with a high mortality rate. We present a case of a 59 -year-old man with a retroperitoneal periaortic mass initially diagnosed as tuberculous aortitis. However following biopsy, the diagnosis was later amended to idiopathic retroperitoneal fibrosis with latent tuberculosis. He was successfully treated with prednisone and methotrexate.

KEYWORDS: Tuberculous aortitis, Ormond's disease, idiopathic retroperitoneal fibrosis

DOI: $10.7861 /$ clinmed.2020-0288

\section{Case presentation}

A 59-year-old man with a known infrarenal abdominal aortic aneurysm and significant smoking history, presented with a 3-month history of dry cough, lethargy, night sweats and $4 \mathrm{~kg}$ weight loss. He was pyrexial on admission. Physical examination was unremarkable. Laboratory tests demonstrated an elevated C-reactive protein $(42 \mathrm{mg} / \mathrm{L}$ ), creatinine of $152 \mu \mathrm{mol} / \mathrm{L}$ (baseline 80), QuantiFERON-TB Gold was positive and serum immunoglobulin G4 (IgG4) was elevated at $2.6 \mathrm{~g} / \mathrm{L}$ (range 0.03-2.01). Whole body computed tomography showed a retroperitoneal mass extending around the infrarenal abdominal aorta (Fig 1), obstructing the right ureter with hydronephrosis, lymphadenopathy adjacent to the mass and a number of lung granulomas. Core biopsy of the mass demonstrated a mixed inflammatory infiltrate comprising macrophages, plasma cells, lymphocytes, neutrophils and a small number of eosinophils, with no evidence of lymphoma, IgG or sclerosing disease. A provisional diagnosis of tuberculous aortitis (TA) was made and he was started on isoniazid, rifampicin, pyrazinamide, ethambutol and $60 \mathrm{mg}$ of

Authors: ${ }^{A}$ consultant in general, geriatric and stroke medicine, MidCentral DHB, Palmerston North, New Zealand; ${ }^{B}$ advanced trainee in neurology and internal medicine, MidCentral DHB, Palmerston North, New Zealand; ${ }^{C}$ consultant physician and rheumatologist, MidCentral DHB, Palmerston North, New Zealand

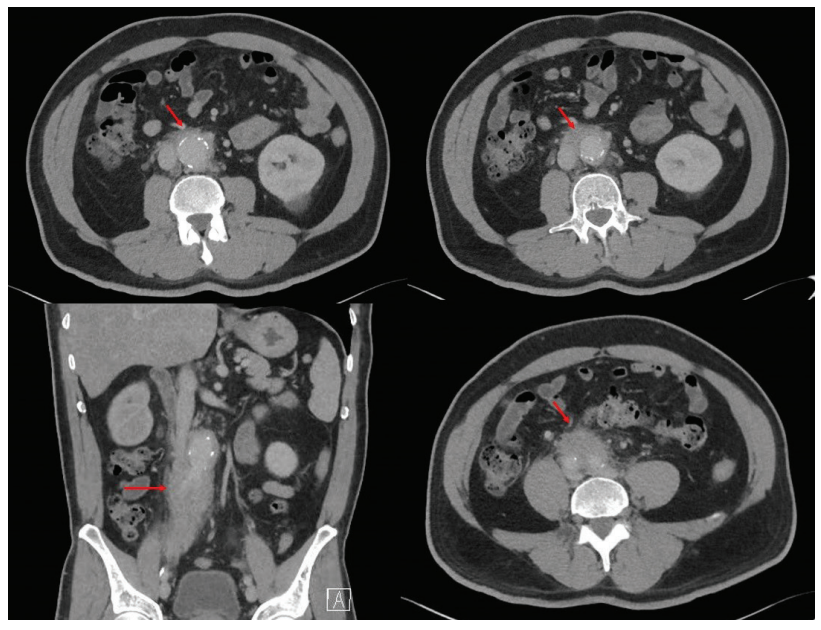

Fig 1. Unenhanced computed tomography of the abdomen demonstrating periaortic mass involving the abdominal aorta and iliac arteries.

prednisone (dosed at $1 \mathrm{mg} / \mathrm{kg} /$ day). A ureteric stent was inserted to relieve the right renal tract obstruction. Treatment led to a resolution of his constitutional symptoms, reduction in C-reactive protein and creatinine, and size of the para-aortic mass on imaging at 6 weeks. Two months later, the biopsy culture was negative and so the diagnosis was thought to be more in keeping with idiopathic retroperitoneal fibrosis (IRPF), also known as Ormond's disease. IgG4 disease was felt to be unlikely, as there were no features of IgG4-related disease on the biopsy. The patient completed 4 months of antituberculous drugs for latent tuberculosis. Methotrexate (20 mg weekly) was started for treatment of IRPF and the prednisone was weaned by $5 \mathrm{mg}$ every month.

\section{Discussion}

Causes of retroperitoneal periaortic masses include infection (eg histoplasmosis, TA), malignancy (eg lymphoma), sarcoidosis, previous radiotherapy and previous surgery. ${ }^{1}$ IRPF is a rare disease, tending to affect males in their fifth decade. The adventitia of the abdominal aorta, iliac arteries and, less commonly, the thoracic aorta can be involved. Symptoms include fevers, weight loss, and pain in the flank, back or abdomen. Ureteral involvement due to ureteral deviation or obstruction is a common complication. $\mathrm{C}$-reactive protein and erythrocyte sedimentation rate is 
usually elevated and can be used to monitor disease activity. Pathologically, the inflammatory infiltrate consists of lymphocytes, plasma cells and macrophages. First-line therapy is glucocorticoid, dosed at $0.75-1 \mathrm{mg} / \mathrm{kg} /$ day with gradual tapering to $5-7.5 \mathrm{mg} /$ day over 6-9 months. Response is rapid with remission rates of $75-95 \%$ after glucocorticoid therapy. ${ }^{2}$ Cyclophosphamide, mycophenolate mofetil and methotrexate have been used effectively as well. Relapse is common and occurs in up to $72 \%$, therefore monitoring with laboratory markers and imaging is required. ${ }^{3}$

TA should also be considered as a differential diagnosis as it carries a mortality rate of up to $50 \%{ }^{4}$. It is often difficult to diagnose due to possible inconclusive culture, histology and blood markers. Treatment is with oral antituberculous drugs for 6 months although surgery may sometimes be required.

\section{Conclusion}

IRPF presents with constitutional symptoms and is a rare cause of retroperitoneal fibrosis. TA should also be considered in the presence of high-risk features, such as immigration/travel from an tuberculosis endemic area, immunosuppression etc as it is associated with significant morbidity and mortality. Both IRPF and TA can be effectively treated. .

\section{References}

1 Tzou M, Gazeley DJ, Mason PJ. Retroperitoneal fibrosis. Vasc Med 2014:19:407-14.

2 Liu H, Zhang G, Niu Y, Jiang N, Xiao W. Retroperitoneal fibrosis: a clinical and outcome analysis of 58 cases and review of literature. Rheumatol Int 2014;34:1665-70.

3 Vaglio A, Maritati F. Idipathic retroperitoneal fibrosis. J Am Soc Nephrol 2016;27:1880-9.

4 Allins AD, Wagner WH, Cossman DV, Gold RN, Hiatt JR. Tuberculous infection of the descending thoracic and abdominal aorta: case report and literature review. Ann Vasc Surg 1999;13:439-44.

Address for correspondence: Dr Karim M Mahawish, Department of Internal Medicine, MidCentral DHB, 50 Ruahine Street, Roslyn, Palmerston North 4442, New Zealand. Email:kmahawish@doctors.org.uk

\section{A new Clinical Medicine and} Future Healthcare Journal subject collection

Articles relating to the COVID-19 pandemic are now gathered together in one handy online subject collection for you to browse.

Browse the collection: www.rcpjournals.org/covid-19 